\title{
A Review of Contact Tracing Approaches for Controlling COVID-19 Pandemic
}

By Md. Tanvir Rahman, Taslima Ferdaus Shuva, Risala Tasin Khan \& Mostofa Kamal Nasir

Mawlana Bhashani Science and Technology University Abstract- The year 2020 will always be in the history of mankind due to the deadly outbreak of COVID-19. Many people are already infected around the world due to the spreading of this novel coronavirus. The virus mainly replicates through close contacts, so there are no other alternatives than to keep social distance, use proper safety gear, and maintain self-quarantine. As a result, the growth of the virus has changed the lifestyle of every individual to a great extent. It is also compelling the Governments to dictate strict lock-downs of the highly affected areas, impose work-from-home approaches where applicable, enforce strict social distancing standards, and so on. Some of the countries are also using smartphonebased applications for contact tracing to track the possibly infected individuals. However, there is a lot of discussion around the world about these contact tracing applications and also about their architecture, attribute, data privacy, and so on. In this paper, we have provided a comprehensive review of these contact tracing approaches in terms of their system architecture, key attributes, and data privacy. We have also outlined a list of potential research directions that can improvise the tracing performance while maintaining the privacy of the user to a great extent.

Index Terms: corona virus, contact tracing, pandemic.

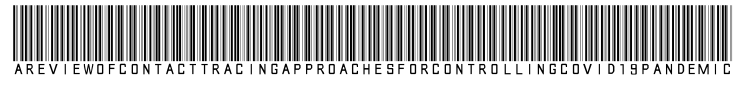

Strictly as per the compliance and regulations of:

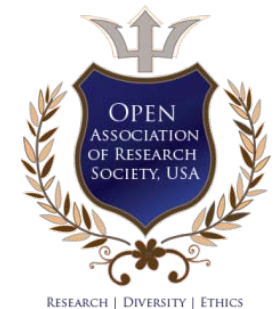

(c) 2021. Md. Tanvir Rahman, Taslima Ferdaus Shuva, Risala Tasin Khan \& Mostofa Kamal Nasir. This is a research/review paper, distributed under the terms of the Creative Commons Attribution-Noncommercial 3.0 Unported License http://creativecommons.org/licenses/by-nc/3.0/), permitting all non-commercial use, distribution, and reproduction in any medium, provided the original work is properly cited. 


\title{
A Review of Contact Tracing Approaches for Controlling COVID-19 Pandemic
}

\author{
Md. Tanvir Rahman ${ }^{\alpha}$, Taslima Ferdaus Shuva ${ }^{\sigma}$, Risala $\operatorname{Tasin} \operatorname{Khan}^{\rho}$ \& Mostofa Kamal Nasir ${ }^{\omega}$
}

\begin{abstract}
The year 2020 will always be in the history of mankind due to the deadly outbreak of COVID-19. Many people are already infected around the world due to the spreading of this novel coronavirus. The virus mainly replicates through close contacts, so there are no other alternatives than to keep social distance, use proper safety gear, and maintain self-quarantine. As a result, the growth of the virus has changed the lifestyle of every individual to a great extent. It is also compelling the Governments to dictate strict lock-downs of the highly affected areas, impose work-from-home approaches where applicable, enforce strict social distancing standards, and so on. Some of the countries are also using smartphone-based applications for contact tracing to track the possibly infected individuals. However, there is a lot of discussion around the world about these contact tracing applications and also about their architecture, attribute, data privacy, and so on. In this paper, we have provided a comprehensive review of these contact tracing approaches in terms of their system architecture, key attributes, and data privacy. We have also outlined a list of potential research directions that can improvise the tracing performance while maintaining the privacy of the user to a great extent.
\end{abstract}

Index Terms: corona virus, contact tracing, pandemic.

\section{INTRODUCTION}

T he World Health Organization (WHO) has declared COVID-19 as a public health emergency of international concern (PHEIC) on January 30, 2020 $[1,2]$ and also a pandemic on March 11, 2020 [3]. According to the Coronavirus disease (COVID-19) Situation Report (by WHO) on 11 July 2020, the total number of confirmed cases was 12322395 globally and among them, 556335 was deceased [4]. To defend this virus, there should be rapid identification and forced quarantine of the infected persons, determination of every other individual with whom they have had close contacts, and the locations where the infected person has visited in recent days [5]. For that reason, many countries have already developed different contact tracing apps to track infected persons and zones [6].

Author $\alpha$ : Department of Information and Communication Technology, Mawlana Bhashani Science and Technology University, Tangail, Bangladesh.e-mail: sajal.it.ju@gmail.com

Author o: Department of Computer Science and Engineering, Daffodil International University, Dhaka, Bangladesh.

e-mail: shuva.cse@diu.edu.bd

Author $\rho:$ The Institute of Information Technology, Jahangirnagar University, Dhaka, Bangladesh. e-mail: risala@juniv.edu

Author w: The Department of Computer Science and Engineering, Mawlana Bhashani Science and Technology University, Tangail, Bangladesh.e-mail:kamal@mbstu.ac.bd
These apps are designed to counterpart contact tracing by using location data acquired from GPS (Global Positioning System) and Bluetooth sensor [7]. Through these applications, it is possible to detect whether a user has been exposed to any COVID-19 positive person or not. Although Bluetooth based solutions for contact tracing are found alluring and being used in developed countries e.g., Singapore, South Korea, etc., there are some negative impacts as it may hamper one's privacy [8, 9]. Besides, any intruder may impersonate and steal valuable information while using the Bluetooth based application. Here, the above-stated solution requires Smartphones to operate. But according to [10], only $24 \%$ of people in India use a smartphone. As a result, the BLE (Bluetooth Low Energy) based solutions will not be appropriate for a list of countries. To address these limitations, the authors of [11] have proposed a solution where the contact tracing can be accomplished by geo location data from mobile-cellular networks. It is also explained that more than $200 \%$ of probably infected persons can be identified as only the cell phone network will be used to measure the location [11]. The only limitation of the approach is that the people have to carry the cell phone with them. Besides these contact tracing apps, few countries like China, France, and South Korea are thinking about the CCTV surveillance technology with image processing. According to Reuters, China has already given an unprecedented glimpse into how to extensively use surveillance cameras to check people's movement [12]. In France, when the lockdown was eased, they monitored every individual by video surveillance cameras [13]. South Korea has been widely admired for its management of the outbreak and spread of coronavirus disease. In South Korean cities, there are over 8 million closed-circuit cameras and that is one camera per 6.3 people [14]. These cameras were heavily used to track the persons who came in near proximity to the infected person. CCTV cameras can work as an investigation tool for detecting various types of content and events, including motion detection, facial recognition, crowd, and so on [15]. The footage can be used for 'video analytics' by which the contact tracing technique will be more convenient [16]. Apart from these approaches, in some of the countries QR code is used as a medium of contact tracing. People are encouraged to keep the unique $\mathrm{QR}$ code with them all the time. Whenever a person is using any public property, s/he is 
scanning the QR code first and by doing so it becomes easy to track every individual.

According to the different proposed approaches and implementations by a list of countries, there are mainly three technological aspects that cover the domain of contact tracing applications. These aspects are BLE (Bluetooth Low Energy) based approach, Geo-location-based approach, and QR Code based approach. In this paper, we have provided a detailed comprehensive review of these approaches in terms of their architecture, feature, and the privacy of the user.

The rest of the paper is organized as follows: Section II emphasizes the review of Contact Tracing applications used by different countries, section III

focuses on the classification of the contact tracing applications, section IV shows the comparison among the different contact tracing approaches, and the conclusion and future work is discussed in section $\mathrm{V}$.

\section{Contact Tracing apps used by Different Countries}

With the alarming spread of COVID-19, researchers around the world are rushing to develop new methods, applications, services, or systems for contact tracing [17]. The purpose of these applications is to identify and notify the persons who were in close contact with a COVID-19 carrier. As a result, many countries are using different contact tracing applications for the safety of the inhabitants. The details of the contact tracing application implemented by a list of the countries around the world are outlined as follows:

\section{a) Singapore}

The government of Singapore has released a mobile phone application titled "TraceTogether" to assist health officials to track down their exposures after an infected individual is identified [18]. The working principle of the application is very simple. The application mainly shares a code to nearby devices where the same app is installed. Both of the devices store the encrypted code in it. When the two users pass by, the application uses the Bluetooth Relative Signal Strength Indicator (RSSI) readings between the devices to estimate the closeness and duration of the meeting. These acquired data (proximity and duration information) are stored on both of the users' phone for 21 days. If a user is found COVID-19 positive, the activity and contact log for the last 14 days are analyzed. Singaporean government made it compulsory to install this app which results in an installation of about $17 \%$ of their total population [19]. As this app uses Bluetooth based approach to operate, it required public acceptance as there were privacy-related issues [20].

\section{b) China}

China has also launched an application titled "Health Code" [21]. The app collects several information about the user such as work address, residential information, contact number, passport number, national identity number, symptom, travel history, and so on. Once submitting the required data, verification will be done via the 'QR Code' which will be sent to the mobile phone. The QR Code can contain either red, green, or amber color code, and depending on the color code the user will be considered risky or risk-free. Users with red color code will be considered risky and will undergo government quarantine or self-quarantine for 14 days, users with amber code will go to quarantine for 7 days but users with green code are considered to be riskfree. The main drawback of this app is if a person intentionally provides wrong information about traveling history or symptoms, he/she might get a green code and affect more people [21].

\section{c) Canada}

For contact tracing, Canada has adopted a "test, trace, and isolate" strategy [22]. In Canada, a contact tracing app named "ABTraceTogether" was launched by the Province of Alberta on May 1, 2020, [23]. Users can voluntarily download the app for tracing and notifying users who may have been exposed to COVID-19 carrier. The public health officials of Alberta Health and Alberta Health Services (AHS) use this application to supplement manual contact tracing. "ABTraceTogether" is also a Bluetooth technologybased application which tracks user's mobile device. It generates an anonymous log of other app users with whom the device user has been in contact. Here the mobile devices exchange Bluetooth-enabled secure encrypted tokens when they are in close contact. The logged data that is collected by Bluetooth proximity is anonymized and encrypted, and does not reveal users' identity [23].

\section{d) South Korea}

South Korea is one of the fewest counties in the world that has almost defeated COVID-19 most effectively. In South Korea, the first confirmed cases were reported on 20th January 2020 and within March 6, 2020, the number of deceased was 42 including 6284 cases which indicates a flattening in the curve of affected and death [24]. For controlling the spread, the Government of South Korea imposed mandatory quarantine to the travelers who departed from other countries in recent times. During their quarantine state, the travelers were forced to install and use a selfdiagnosis app through which they updated their health status regularly so that the Government can get informed whether any of them is a potential carrier of COVID-19 or not [25]. The drawback of this tracing process in violation of private data as the collected data were shared among many authorities such as police, health insurance, government agencies, health care professionals, and others [26]. 


\section{e) Australia}

The Australian Federal Government launched a contact tracing application titled "COVIDSafe" on April 26, 2020 [27]. Like previous applications, it also uses Bluetooth technology to record contact between users. Although it has shown a significant effect on tracing COVID-19 positive people in Australia it is already facing a lot of debate because of its transparency and privacy issues [27].

\section{f) France}

Like many European countries, the French government introduced a contact tracing app, titled "StopCovid". It works using Bluetooth technology and provides data privacy, protection, and transparency [28].

\section{g) Germany}

The German Federal government has launched a "BLE" (Bluetooth Low Energy technology) based application "Corona-Warn-App" on June 16, 2020 [29]. There are no major privacy concerns as the-App has been designed with a special focus on privacy from the beginning.

\section{h) Indonesia}

Indonesian Ministry of Information and Communication $(\mathrm{MOCl})$ launched a mobile application called "PeduliLindungi" which uses Bluetooth and GPS (Global Positioning System) both. Users register and share their locations during their traveling. This app traces whether they were in contact with COVID-19 patients or not. While it traces someone entering crowds whom they are calling "COVID-19 red zones", the application alerts the user [30].

\section{i) Poland}

The Polish Government has launched two applications titled "Kwarantanna domowa" and "ProteGO Safe Safe". The "Kwarantanna domowa" uses geolocation and face recognition technology to ensure the quarantine of relevant people. The app is designed to track whether COVID-19 patients are in quarantine or roaming around. The app will time to time ask for selfies from the app user to ensure his location and thus using image processing and geological data will ensure spreading the virus by limiting patients' movement [31]. Poland govt. using another app "ProteGO Safe Safe", which uses Bluetooth short-range radio. This technology is also being used by Apple and Google to securely exchange keys among the smartphones who have been near to each other [32].

\section{j) South Africa}

For tracking COVID-19 patients South African app Covi-ID has worked with a different approach by using QR codes. The working principle of the app is providing each user with a QR code after his/her registration in the app. While registering the app, the user needs to provide his information on being COVID19 positive or negative. He/she then get a QR code that needs to be scanned while he/she travels by vehicles or enters any public place like shopping malls, educational institutions, etc. Whenever the user gets to know about his being COVID-19 positive he updates the status in the app and it alerts all the vehicles and places he visited previously [33]. Thus, each time the QR code is being scanned the geo-location of the user can be located for further tracking the individual. Their identification is being checked using blockchain [34].

\section{k) India}

The Indian government has made it compulsory to install an app named "Aarogya Setu" for the government employees to control the spread of COVID19 [35]. This application uses BLE (Blue-tooth Low Energy) and GPS (Global Positioning System) both for tracking COVID-19 infections. By Bluetooth, it checks whether a user has been exposed to (within six feet of) any COVID-19 positive patients or not. And using geological location information, the app determines whether the current location of the user belongs to one of the infected areas or not.

\section{1) Switzerland}

"Swisscovid" is the most popular app for contact tracing in Switzerland. It is also a Bluetooth based application which needs smartphones with the users to track COVID-19. While two devices come in close contact, they exchange random IDs that remain on the phone for the next 14 days before automatically delete. It is more likely the other BLE based apps like German's "Corona-Warn-App" or France's StopCovid" [36]. Like lots of BLE-based other applications, it was accused of a large set of problems including falsepositive attack, cryptography weakness, and so on [37].

\section{m) Pakistan}

Under the supervision of the Ministry of IT and Telecommunication, the Pakistan government has developed an app named "COVID-19 Gov PK" for raising awareness among citizens about COVID-19. The app needs to access the user's geo-location during the installation time of installation [38].

Netherlands, Turkey, UAE, UK, U.S. also using different contact tracing apps for controlling the spread of COVID-19. So, from the described scenario, we can conclude in a point that most of the countries around the world are using applications that are mainly BLE (Bluetooth Low Energy) GPS (Global Positioning System) based.

\section{ini. Classification of Contact Tracing Applications}

Numerous contact tracing applications are already implemented in some of the countries around the world and some others are proposed by the 
researchers. Most of these applications are smartphone-based which require Bluetooth while some other approaches do not require any smartphone at all rather any cellphone would work. Even in some scenarios, the QR code is considered to be the only solution. As a result, the applications can be classified or categorized based on some technical aspects as follows:
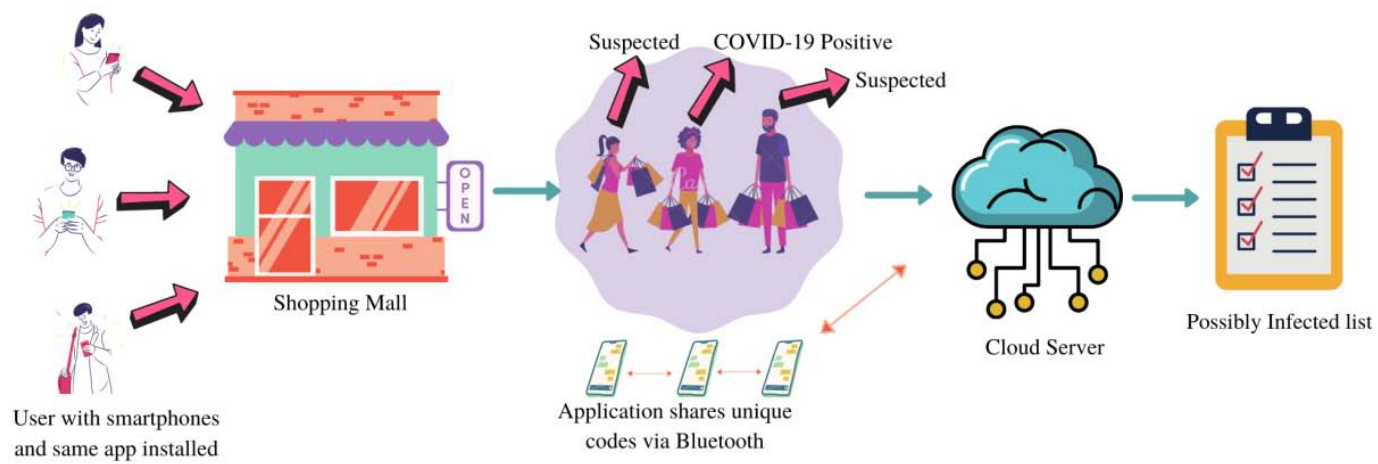

Fig. 1: Summarized steps in Bluetooth based Contact Tracing approach

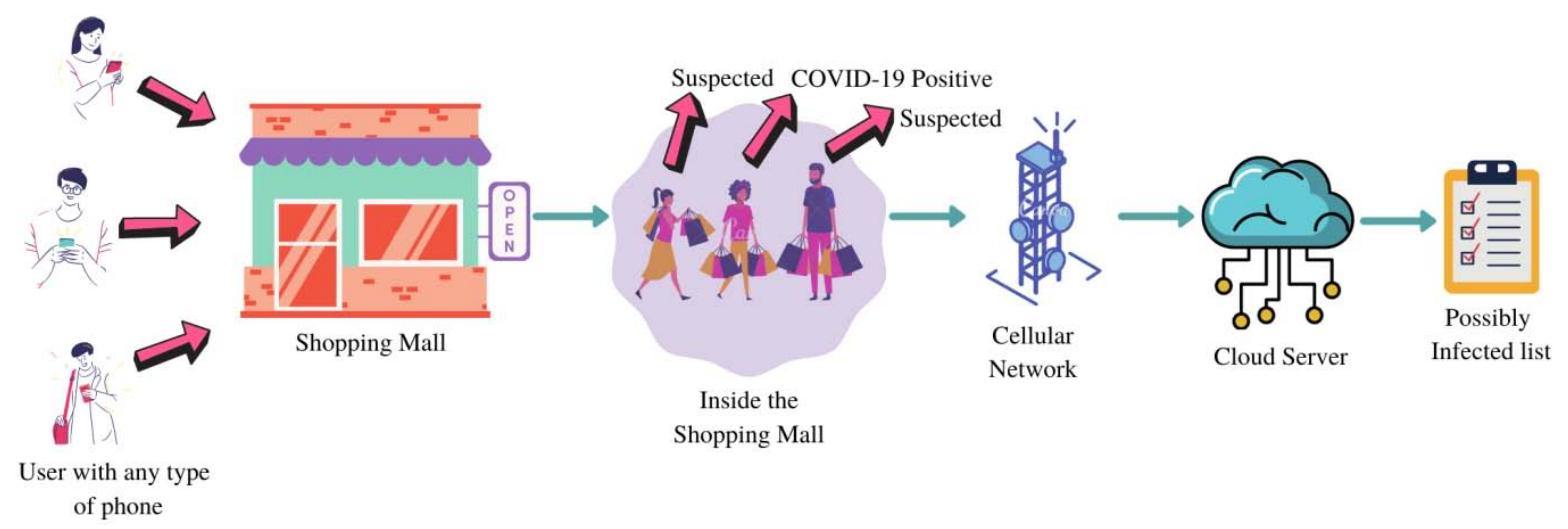

Fig. 2: Summarized steps in Geo Location based Contact Tracing approach

a) Bluetooth Based Approach

In Bluetooth based approach, firstly the user has to install the application. Then if the user passes at near proximity with a COVID-19 carrier (who also had installed the same application previously on his/her phone), the application can record the data and show the intensity of risk. The summarized steps are as follows:

- Step 01: Miss. A, Mr. B, Mr. C, and so on installs the application and keeps the Bluetooth turned on

- Step 02: Miss. A, Mr. B and Mr. C visit the nearest shop

- Step 03: The applications share unique codes when Miss. A, Mr. B, and Mr. C were in close proximity

- Step 04: Mr. B is tested as COVID-19 positive

- Step 05: Miss. A and Mr. C is on the suspected list

- Step 06: Any application user can check whether he or she was in close proximity with any COVID-19 carrier or not

Fig. 1 shows the summarized steps of Bluetooth based Contact Tracing approach. From the figure, a list of people is going to a shopping mall. But previously all of them have installed the contact tracing application on their smartphone. All of them are also keeping the Bluetooth of their smartphone turned on. Now, at any point inside the shop, they were in close proximity to one another. At that time, the application shares a unique code with one another. These data are also synchronized with the server. Later on, it is found that one of them is COVID-19 positive. As a result, the other two people also lie in the suspected list. Here, the suspected list was generated with the help of a shared code by Bluetooth.

\section{b) Geo-location Based Approach}

In the Geo-location-based approach, the location data of the COVID-19 carrier will be collected from the SIM (Subscriber Identity Module) operator rather than any installed application. The main advantage is that to trace the COVID-19 carrier, no user application is needed. But in this approach active government support is required. The summarized steps are as follows: 
- Step 01: Miss. A, Mr. B, and Mr. C visit the nearest shop with their mobile phone (any type of mobile phone that is connected to a cellular network)

- Step 02: Mr. B is COVID-19 positive

- Step 03: Miss. A and Mr. C is on the suspected list

- Step 04: The location points (latitude and longitude) of Mr. B for the last 7 days are also collected from the cellphone operator.

- Step 05: These location points are considered as red points

- Step 06: The red points are sent to all other cellphone operators to collect the list of all possibly infected individuals.
Fig. 2 illustrates the summarized steps of the cellphone network-based Contact Tracing approach. From the figure, a list of people is going to a shopping mall. But in this approach, the end-user does not need to install any kind of application in his/her phone and even the phone does not need to be a smartphone rather any kind of mobile phone with an active SIM card will work. Here, the location data of the user will be collected from the cell phone network and synchronized with the server.

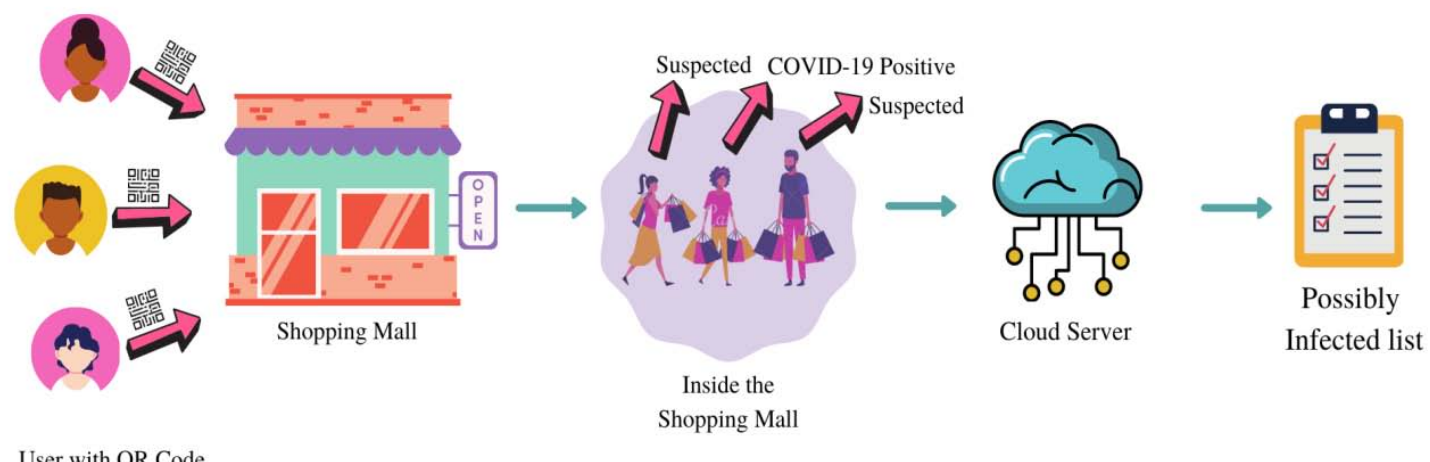

Fig. 3: Summarized steps in QR Code based Contact Tracing approach

c) QR Code Based Approach

In this approach, the people are monitored via QR code. People are instructed to keep a unique QR code with them all the time for example when a user is using a public bus or entering a shopping mall and so on. in this approach, it is also instructed that even if the user has no smartphone, the QR code must be printed in hard copies. QR code scanner will be everywhere so that people can scan their QR code and do the necessary things. The summarized steps are as follows:

- Step 01: Miss. A, Mr. B, Mr. C, and so on acquires the QR code from the authority

- Step 02: Miss. A, Mr. B, and Mr. C visit the nearest shop by scanning the QR code

- Mr. B is tested as COVID-19 positive

- Miss A and Mr. C is on the suspected list

- Any application user can check whether he or she was in close proximity with any COVID-19 carrier or not
Fig.3 shows the summarized steps of the QR code-based Contact Tracing approach. From the figure, a list of people is going to a shopping mall. Before that, all of them has got their unique $\mathrm{QR}$ code from the authority. These QR codes will be used when any person will use any public property for example public bus, public toilet, etc. Before entering the shopping mall, all of them have shown the QR code to the scanner. If any person at that shopping mall was found COVID-19 positive, then all others will be at risk.

\section{Comparative Analysis}

At this point, we want to provide a comprehensive analysis of the strengths and limitations of Bluetooth based Contact Tracing, Geo-location based Contact Tracing, and QR Code based Contact Tracing as follows:

Table 1: Comparative Study of Strength and Limitation of Different Contact Tracing Approaches

\begin{tabular}{|c|l|l|}
\hline Approach & \multicolumn{1}{|c|}{ Major Strength } & \multicolumn{1}{c|}{ Major Limitation } \\
\hline & $\begin{array}{l}\text { - Bluetooth operates effectively at at } \\
\text { close range } \\
\text { - Anonymous code is used for each } \\
\text { device } \\
\text { B No cellular data is needed for the } \\
\text { application to operate }\end{array}$ & $\begin{array}{l}\text { - The person has to carry a phone } \\
\text { The phone must be a smartphone } \\
\text { with a Bluetooth sensor } \\
\text { - User's privacy may be hampered } \\
\text { as any unwanted access can be } \\
\text { possible through Bluetooth } \\
\text { - The user is bound to install the } \\
\text { application hence or otherwise the }\end{array}$ \\
\hline
\end{tabular}




\begin{tabular}{|c|c|c|}
\hline & & $\begin{array}{l}\text { approach won't work } \\
\text { - This approach can generate false- } \\
\text { positive results. For example, there } \\
\text { can be a wall in between two } \\
\text { persons inside a building. }\end{array}$ \\
\hline Geolocation Based & $\begin{array}{l}\text { - The mobile phone of the user can } \\
\text { be of any type. For example, it can } \\
\text { be a smartphone or it can be a very } \\
\text { simple button phone with no touch } \\
\text { screen and Bluetooth and other } \\
\text { sensors } \\
\text { - Mass people do not need to install } \\
\text { any type of application } \\
\text { - There is no chance that the } \\
\text { location data is inaccurate if the } \\
\text { real user of the mobile is carrying it } \\
\text { - Less amount of false negative } \\
\text { issue }\end{array}$ & $\begin{array}{l}\text { - The person has to carry the } \\
\text { cellphone } \\
\text { - If someone carries other's } \\
\text { cellphone, then this process won't } \\
\text { work } \\
\text { - At different levels of a building, the } \\
\text { geo-location approach detects the } \\
\text { location points as the same }\end{array}$ \\
\hline QR Code Based & $\begin{array}{l}\text { - Appropriate for poor countries } \\
\text { where people have limited access } \\
\text { to mobile phones (Printed QR } \\
\text { Codes) } \\
\text { - There is no technological } \\
\text { dependency. Any type of person } \\
\text { can use it. } \\
\text { - From the users perspective, it } \\
\text { works in an offline perspective }\end{array}$ & $\begin{array}{l}\text { - May produce false-positive results } \\
\text { while determining close contact } \\
\text { among infected and healthy } \\
\text { individuals } \\
\text { - This approach is fully dependent } \\
\text { on the will of the mass people as } \\
\text { the physical distance is not } \\
\text { measured in the process rather the } \\
\text { process-aware the people about } \\
\text { the fact }\end{array}$ \\
\hline
\end{tabular}

From Table 1, it is clear that none of the approaches is bullet-proof. Each of the approaches has its strengths and limitations. From the users' perspective, there will be some privacy-related issues for each of the contact tracing approaches. For the Bluetooth based approach, it is highly probable that any intruder may gain some improper access to the device of a person and steal valuable information which is irrecoverable. Although the Bluetooth based approach shares encrypted code among devices, even then it may run into some attack through Bluetooth. On the other hand, when the location data of the person is collected from the cellular network, it is less attack prone. So, the data privacy of the user is preserved in the Geo-location-based approach when compared to Bluetooth based Contact Tracing. Lastly, the QR code is applicable to the under-developed countries where very limited people have mobile phones. In this approach, it is instructed that the person should keep the QR code all the time if the soft copy is not possible then in hard copies. So, there is no chance of privacy-related issues for the people.

\section{Conclusion}

The novel Coronavirus can only be controlled via safety measures. Contact Tracing approaches are one of the most important and beneficial precautions that can be utilized to trace the spread of the disease. It is expected that most of the adults around the world have a mobile phone of any type. In our research, we have found that all of the implemented and proposed contact tracing approaches can be categorized into three segments based on technological aspects such as Bluetooth based Contact Tracing, Geo-location based Contact Tracing, and QR code-based Contact Tracing. We can obviously predict that these available contact tracing approaches can play a vital role in defeating the pandemic. We have also analyzed the approaches and found that there should be more research related to platform-independent contact tracing, there can be any physical device or sensor for this inside the smartphone or wearable. Apart from hardware changes, researchers may focus on Artificial Intelligence-based algorithms for contact tracing solutions.

\section{References Références Referencias}

1. Farhana K M (2020), Knowledge and Perception towards Novel Coronavirus (COVID-19) in Bangladesh. SSRN. https://dx.doi.org/10.2139/ ssrn.3578477

2. WHO (2020) Statement on the second meeting of the International Health Regulations (2005) Emergency Committee regarding the outbreak of novel coronavirus (2019-nCoV). https://www.who. int/news/item/30-01-2020-statement-on-the-secondmeeting-of-the-international-health-regulations-(20 05)-emergency-committee-regarding-the-outbreakof-novel-coronavirus-(2019-ncov). Accessed 27 November 2020. 
3. WHO (2020) WHO Director-General's opening remarks at the media briefing on COVID-19. https://www.who.int/dg/speeches/detail/who-direct or-general-s-opening-remarks-at-the-media-briefing -on-covid-19---11-march-2020. Accessed 27 November 2020.

4. WHO (2020) Coronavirus disease (COVID-19) Weekly Epidemiological Update and Weekly Operational Update. https://www.who.int/emerg encies/diseases/novel-coronavirus-2019/situationreports. Accessed 27 November 2020.

5. Raskar $\mathrm{R}$ et al (2020) Apps gone rogue: Maintaining personal privacy in an epidemic. arXiv. https://arxiv.org/abs/2003.08567

6. Ahmed $\mathrm{N}$ et al (2020) A Survey of COVID-19 Contact Tracing Apps. IEEE Access. 8:134577134601. https://doi.org/10.1109/ACCESS.2020.301 0226

7. Chan J et al (2020) Pact: Privacy sensitive protocols and mechanisms for mobile contact tracing. arXiv. https://arxiv.org/abs/2004.03544

8. Sonn J W (2020) Coronavirus: South Korea's success in controlling disease is due to its acceptance of surveillance. https://theconversation. com/coronavirus-south-koreas-success-in-controll ing-disease-is-due-to-its-acceptance-of-surveillance -134068. Accessed 27 November 2020

9. Kaptchuk $\mathrm{G}$ et al (2020) How good is good enough for COVID19 apps? The influence of benefits, accuracy, and privacy on willingness to adopt. arXiv. https://arxiv.org/abs/2005.04343

10. Statista (2020) Smartphone ownership rate by country 2019. https://www.statista.com/statistics/ 539395/smartphone-penetration-worldwide-bycountry/. Accessed 27 November 2020.

11. Rahman M T et al (2020) An Automated Contact Tracing Approach for Controlling Covid-19 Spread Based on Geolocation Data from Mobile Cellular Networks. IEEE Access https://doi.org/10.1109/ ACCESS.2020.3040198

12. Cadell C (2020) China's coronavirus campaign offers glimpse into surveillance system. https://www. reuters.com/article/us-health-coronavirus-china-surv eillance/chinas-coronavirus-campaign-offers-glimp se-into-surveillance-system-idUSKBN2320LZ. Accessed 27 November 2020.

13. BBC (2020) Coronavirus France: Cameras to monitor masks and social distancing. https://www. bbc.com/news/world-europe-52529981. Accessed 27 November 2020.

14. Zastrow M (2020) Coronavirus contact-tracing apps: can they slow the spread of COVID-19? https://www.nature.com/articles/d41586-020-015142. Accessed 27 November 2020.

15. O'dea $S$ (2020) Number of smartphone users from 2016 to 2021. https://www.statista.com/statistics/ 330695/number-of-smartphone-users-worldwide/.

Accessed 27 November 2020.

16. Ashby M (2017) The Value of CCTV Surveillance Cameras as an Investigative Tool: An Empirical Analysis. European Journal on Criminal Policy and Research. 23:441-459. https://doi.org/10.1007/s 10610-017-9341-6

17. O'Neill P H (2020) A flood of coronavirus apps are tracking us. Now it's time to keep track of them. https://www.technologyreview.com/2020/05/07/100 0961/launching-mittr-covid-tracing-tracker/.

Accessed 27 November 2020.

18. Cho H et al (2020) Contact Tracing Mobile Apps for COVID-19: Privacy Considerations and Related Trade-offs. arXiv. https://arxiv.org/abs/2003.11511

19. Bay $J$ et al (2020) Bluetrace: A Privacy-Preserving Protocol Forcommunity-Driven Contact Tracing Across Borders. https://bluetrace.io/static/ bluetrace_whitepaper-938063656596c104632def38 3eb33b3c.pdf

20. Trivedi A et al (2020) WiFiTrace: Network-based Contact Tracing for Infectious Diseases Using Passive WiFi Sensing. arXiv. https://arxiv.org/abs/2005.12045

21. Hua J \& Shaw R (2020) Corona Virus (COVID-19) "Infodemic" and Emerging Issues through a Data Lens: The Case of China. Int. J. Environ. Res. Public Health. 17:2309. https://doi.org/10.3390/ijerph1707 2309

22. Austin L M et al (2020) Test, Trace, and Isolate: COVID-19 and the Canadian Constitution. Osgoode Legal Studies Research Paper. https://ssrn.com/ abstract $=3608823$ or http://dx.doi.org/10.2139/ ssrn. 3608823

23. Alberta (2020) AB Trace Together. https://www. alberta.ca/ab-trace-together.aspx. Accessed 27 November, 2020.

24. Shim E et al (2020) Transmission potential and severity of COVID-19 in South Korea. International Journal of Infectious Diseases. 93: 339-344. https://doi.org/10.1016/j.ijid.2020.03.031.

25. $\mathrm{Ko} \mathrm{H}$ et al (2017) Structure and enforcement of data privacy law in South Korea. International Data Privacy Law. 7(2):100-114. https://doi.org/10.1093/ idpl/ipx004

26. Rousan N A \& Najjar H A (2020) Data Analysis of Coronavirus CoVID-19 Epidemic in South Korea Based on Recovered and Death Cases. Journal of Medical Virology. https://doi.org/10.1002/jmv.25850

27. Greenleaf G and Kemp K (2020) Australia's 'COVIDSafe App': An Experiment in Surveillance, Trust and Law. University of New South Wales Law Research Series 999. https://ssrn.com/abstract= 3589317

28. Dillet $R$ (2020) France releases contact-tracing app StopCovid | TechCrunch. https://techcrunch.com/ 2020/06/02/france-releases-contact-tracing-app- 
stopcovid-on-android/. Accessed 27 November 2020.

29. Made for Mind (2020) Loved or loathed? How Germany's coronavirus tracking app is faring. https://www.dw.com/en/loved-or-loathed-howgermanys-coronavirus-tracking-app-is-faring/a53959165. Accessed 27 November 2020.

30. Abrahams $\mathrm{N}$ et al (2020) Contact tracing apps: A new world for data privacy. https://www.norton rosefulbright.com/en-us/knowledge/publications/d7 a9a296/contact-tracing-apps-a-new-world-for-dataprivacy. Accessed 27 November 2020.

31. Bartoszko A (2020) Accelerating Curve of Anxiousness: How a Governmental Quarantine-App Feeds Society with Bugs. Journal of Extreme Anthropology. 4(1):E7-E17 https://doi.org/10.5617/ jea.7861

32. Koper A (2020) Poland rolls out privacy-secure coronavirus tracking app. https://www.reuters.com/ article/us-health-coronavirus-poland-tech/polandrolls-out-privacy-secure-coronavirus-tracking-appidUSKBN23G208. Accessed 27 November 2020.

33. Kriesch A (2020) South African app aims to slow spread of COVID-19 in developing nations. https:// www.dw.com/en/south-african-app-aims-to-slow-sp read-of-covid-19-in-developing-nations/a-534473 46. Accessed 27 November 2020.

34. Sawant N (2020) AAROGYA SETU: whether we like it or not, the app is here to stay, but it's still riddled with privacy issues that need strong answers. https://www.firstpost.com/tech/news-analysis/aaro gya-setu-whether-we-like-it-or-not-the-app-is-hereto-stay-but-its-still-riddled-with-privacy-issues-thatneed-strong-answers-8348131.html. Accessed 27 November 2020.

35. FOPH (2020) Coronavirus: SwissCovid app and contact tracing. https://www.bag.admin.ch/bag/en/ home/krankheiten/ausbrueche-epidemien-pandem ien/aktuelle-ausbrueche-epidemien/novel-cov/swis scovid-app-und-contact-tracing.html. Accessed 27 November 2020.

36. Dehaye P. \& Reardon J. (2020) SwissCovid: a critical analysis of risk assessment by Swiss authorities. arXiv. https://arxiv.org/abs/2006.10719

37. Moitt-PK (2020) Application developed to deal with coronavirus. https://moitt.gov.pk/NewsDetail/NjQ3 NWQyMDMtYTBIYy00ZWEOLWI2YjctYmFmMjk4MTA 1MWQ0. Accessed 27 November 2020.

38. Ministry of Information Technology and Telecommunication, Application Developed to Deal with Coronavirus. https://moitt.gov.pk/NewsDetail/ NjQ3NWQyMDMtYTBIYy00ZWEOLWI2YjctYmFmMjk 4MTA1MWQ0. Accessed 27 November 2020. 